\title{
Geografía Electoral de la Ciudad Autónoma de Buenos Aires, 2015. Elecciones a Jefe de Gobierno municipal y a Presidente de la República Argentina
}

Joaquín Bosque Sendra ${ }^{1}$

Gustavo D. Buzai ${ }^{2}$
Recibido: 19 de enero, 2017

Aceptado: 28 de mayo, 2017

\section{RESUMEN}

El presente trabajo analiza aspectos de la Geografía Electoral de la Ciudad Autónoma de Buenos Aires en el año 2015 a partir de los resultados de las elecciones a Jefe de Gobierno Municipal y a Presidente de la República Argentina. Incluye aspectos teóricos de la Geografía como ciencia que aborda el proceso electoral en su dimensión espacial. El caso de estudio aplica el análisis exploratorio de datos espaciales con la finalidad de analizar las asociaciones entre la distribución espacial del voto, el mapa social y los máximos niveles educativos alcanzados por la población. A partir de la perspectiva geográfica se podrán comprobar hipótesis o avanzar en interpretaciones focalizadas en la espacialidad de los resultados.

\section{Palabras clave | Geografía electoral, análisis espacial electoral, distribución espacial del voto, elecciones argentinas 2015, Ciudad Autónoma de Buenos Aires.}

\footnotetext{
${ }^{1}$ Doctor en Geografía, Departamento de Geología, Geografía y Medio Ambiente, Universidad de Alcalá, España. Correo electrónico: joaquin.bosque@uah.es

2 Doctor en Geografía, Instituto de Investigaciones Geográficas, Universidad Nacional de Luján / CONICET, Argentina. Correo electrónico: buzai@mail.unlu.edu.ar

* Trabajo realizado en el marco del proyecto "La nueva Geografía Aplicada: Sistemas Complejos, Geografía Automatizada y SIG en el estudio de las actuales estructuras socioespaciales". Código: CDDCS-85807. Radicado y financiado por el Departamento de Ciencias Sociales de la Universidad Nacional de Luján (Argentina). Director: Gustavo D. Buzai.
} 


\section{ABSTRACT}

Electoral Geography of the Autonomous Gity of Buenos Aires, 2015. Elections to Head of Municipal Government and to President of the Argentine Republic

The present paper analyzes aspects of the Electoral Geography of Buenos Aires in 2015 based on the results of the elections to Head of Municipal Government and President of the Argentine Republic. It includes theoretical aspects considering Geography as a science that approaches the spatial dimension of electoral process. The case study applies exploratory spatial data analysis in order to analyze the associations between the spatial distribution of voting, the social map of the city and the level of education of the population. From the geographical perspective we will be able to test hypotheses and advance in interpretations focused on the spatiality of the results.

Keywords | Electoral geography, electoral spatial analysis, spatial distribution of vote, argentine elections 2015, Autonomous City of Buenos Aires.

\section{INTRODUCCIÓN}

El 2015 fue un año electoral en la República Argentina. Se llevó a cabo la elección a Presidente de la Nación junto a la elección legislativa para el Congreso Nacional y parlamentarias del Parlasur (Parlamento del Mercosur). En la mayoría de las provincias (21 de 23) hubo elección a Gobernador y en la Ciudad Autónoma de Buenos Aires (CABA) se eligió Jefe de Gobierno. En este contexto, resulta de interés analizar el comportamiento electoral en la CABA, principal ciudad de la República Argentina y distrito que gravita con importante peso político a través de dos gestiones locales consecutivas del partido político Propuesta Republicana (PRO) con el Jefe de Gobierno Mauricio Macri, que con poste rioridad a dos mandatos consecutivos (2007-2011 y 2011-2015) se presenta como candidato en la elección presidencial 2015.

El objetivo de este trabajo consiste en analizar la Geografía Electoral de la CABA en las últimas elecciones a Jefe de Gobierno de la CABA y a Presidente de la República Argentina con la finalidad de comprobar una hipótesis recapitulativa: las distribuciones espaciales del voto son producto del mapa social y de los niveles educativos de la población en el área de estudio.

Se incluyen aspectos teóricos de la Geografía como ciencia que puede lograr una 
focalización espacial del proceso electoral, se sistematizan los datos empíricos de las votaciones y se representan las distribuciones espaciales de los votos obtenidos por cada partido político. Finalmente su asociación espacial con el mapa social y los máximos niveles educativos alcanzados brindará elementos para verificar ciertos efectos de contexto sobre las diferentes preferencias. El análisis exploratorio realizado permite el descubrimiento de los patrones de distribución que presenta la dimensión espacial electoral. A partir de esta base se podrán comprobar hipótesis y avanzar en interpretaciones que se focalicen en la espacialidad de los resultados obtenidos.

\section{GEOGRAFÍA Y PROCESO ELECTORAL}

La Geografía Política estudia aspectos teóricos y prácticos del ejercicio del poder sobre el espacio geográfico, lo cual se plasma concretamente en la organización política del territorio. La Geografía Electoral forma parte de la Geografía Política combinando aspectos relativos a la relación entre las instituciones de gestión y el comportamiento electoral de los ciudadanos. Es una materia no muy cultivada dentro del mundo académico geográfico ya que en cada país son pocos los investigadores que aportan investigaciones y publicaciones sobre el tema.

Su importancia en los años 60/70 del siglo XX se debe a los trabajos de unos pocos autores anglosajones: Cox (1971, 1969 y 1998), Busteed (1975) y Taylor y Johnston (1979), período destacado por Johnston y Pattie (2012). En estos trabajos se aplicaron diferentes procedimientos estadísticos para el análisis de los datos electorales y desarrollaron una teoría de base comportamental (behaviorista) para explicar las preferencias electorales plasmadas a través del votos.

En Argentina los estudios de Geografía Electoral se presentan en aportes discontinuos desarrollados con apoyo del análisis espacial cuantitativo. En esta línea podemos nombrar los aportes de Velázquez (1989) que, en su análisis de las elecciones de 1983, describe los resultados e intenta encontrar alguna explicación social de ellos, para lo que plantea usar el concepto de "polarización"; Medús $(1997,2002)$, en el primer trabajo plantea ideas teóricas sobre la Geografía electoral, mientras que, en el segundo, su tesis doctoral, describe los resultados municipales en la provincia de La Pampa y, finalmente, Monzón (2009a, 
b), discute, en sus dos trabajos, los conceptos e ideas básicas de la Geografía electoral, sin llegar a realizar estudios empíricos.

Debemos tener en cuenta que los resultados electorales pueden resultar de gran interés para la Geografía de base positivista, ya que permiten disponer de miles, millones de datos, muy controlados para que tengan pocos errores, repetidos regularmente, y referidos a un tema de gran interés social. Los académicos de las ciencias sociales en general, han hecho uso de estos datos de manera variada, y es llamativo el limitado uso que ha mostrado la investigación geográfica. Los diferentes temas que suelen estudiarse dentro la Geografía electoral pueden agruparse en las siguientes líneas de análisis:

$1^{\circ}$ La Geografía de las votaciones presenta y describe los resultados electorales, no solo los decisivo (Bosque Sendra, 1988).

$2^{\circ}$ Las campañas electorales resultan ser un tema de estudio poco usual, pero genera interés en la descripción y análisis de las estrategias que los diferentes partidos políticos suelen realizar para atraer el voto del electorado (Bosque Sendra, 1981b; Palma y Morales, 2014).

$3^{\circ}$ La explicación del voto intenta comprender las razones de los resultados producidos en cada elección. En este tema los geógrafos coinciden, y en cierta manera compiten en temáticas que se solapan con el interés de otros científicos sociales. En general, se considera que los factores explicativos tienen relación a un efecto generado por el grupo social o conexiones sociales de pertenencia (enfoque contemplado habitualmente por sociólogos y politólogos) o un efecto espacial o conexiones de vecindad (enfoque empleado habitualmente por los geógrafos) (ver Bosque Sendra, 1980, 1982). En la última década se realizaron aplicaciones de métodos estadísticos multinivel en un análisis conjunto (Jones et al., 1992; Gombin, 2014) lo que supone combinar aspectos a-espaciales y espaciales.

$4^{\circ}$ El estudio y la reforma/mejora de los sistemas electorales enfoca los procedimientos de diverso tipo que intentan resolver la muy compleja tarea de convertir los miles, millones de votos emitidos en la elección para la determinación de unos pocos cientos de escaños. Se debe subrayar que, en general, los sistemas electorales tienen una base territorial muy pronunciada, la definición espacial de las circunscripciones electorales es fundamental en 
el resultado haciéndose evidente el problema de la definición espacial de la unidades espaciales (Gould, 2000; Escandón y López, 2004).

\section{Factores explicativos del voto}

De acuerdo con la mencionada teoría behaviorista de la elecciones, la decisión de voto se adopta, por personas que viven en un contexto espacial, corno resultado de un balance de informaciones sobre las distintas opciones electorales (Cox, 1971). Este balance se produce en base a la circulación de datos e ideas a través de una amplia red en la cual cada elector es un nodo conectado a otros. La pertenencia de cada elector a un grupo social específico con diferentes condiciones de vida, determina que reciba una información más o menos sesgada a favor de ciertos partidos, ya que cada miembro de un grupo social suele tener mayor facilidad de relación y comunicación con personas de ese grupo que con el resto de la sociedad. Se origina una tendencia entre todos los miembros de un grupo social a votar de manera parecida. Se trata del denominado efecto de grupo o de la estructura social, y es un aspecto destacado habitualmente por los análisis sociológicos que relacionan las características del voto con las de los grupos sociales.

Junto a este primer factor explicativo es posible considerar otro que se centra en la dimensión espacial, la Geografía electoral ha encontrado en muchas ocasiones la actuación del denominado efecto de vecindario como responsable del contagio espacial en el voto (Bosque Sendra, 1981a). La explicación se puede formular en estos términos: en un área suelen convivir varios grupos sociales, aunque siempre predomine uno de ellos. Por ello, aunque se produce una interrelación entre las actitudes que podemos llamar «naturales» de cada grupo social, a dar su apoyo a un determinado partido político, el sesgo informativo en el área se orientará hacia la expresión «natural» del grupo social más numeroso.

Esto puede determinar que el voto a ese partido resulte superior a lo que debería ser de acuerdo a la adición de la importancia de su apoyo social natural y los miembros de otros grupos sociales que los votan a partir de la inercia producida por el flujo de informaciones favorables que superan la del resto de los partidos. En síntesis los resultados electorales tienen dos grandes componentes, la acción de la estructura social a través del efecto de grupo y la acción de la dimensión espacial a través del efecto vecindario.

Es posible medir el efecto vecindario si se elimina la influencia que la estructura social tiene en cada zona. Puede realizarse mediante la utilización de análisis de regresión en base a los residuos proporcionados por variables de la estructura 
social, a través de la diferencia entre un comportamiento teórico esperado y el resultado empírico obtenido o a través de medir un efecto de vecindario temporal (EVT) como efecto que podrá existir entre el electorado de un partido en una fecha y la votación a ese partido en una fecha posterior. Esto último se verá posible en el caso de la República Argentina en la cual las elecciones se realizan a partir de dos votaciones (elecciones primarias y generales).

Se puede legítimamente considerar que el electorado que en un momento dado vota a un partido se convierte, a partir de esa fecha, en un agente difusor de informaciones favorables a dicho partido, y de ese modo aumenta su propaganda. Esta tendencia incrementa las posibilidades comunicacionales del partido mayoritario en detrimento de la circulación de noticias sobre otros partidos. De esta manera se verifica la conversión del electorado de los partidos perdedores hacia el partido ganador, de manera que en una siguiente elección el partido mayoritario termina incrementando sus votos.

\section{Área de estudio: Ciudad Autónoma de Buenos Aires}

La Ciudad Autónoma de Buenos Aires (CABA) es la ciudad capital de la República Argentina. Situada sobre la margen derecha del río de la Plata su función portuaria la convirtió históricamente en la principal puerta de entrada y salida del país al concentrar los mayores flujos de productos y población. Tuvo dos fundaciones, la primera fue realizada por Pedro de Mendoza el 3 de febrero de 1536 y la segunda por Juan de Garay el 11 de junio de 1580. El primer asentamiento fue un fuerte que quedó aislado ante el asedio de las poblaciones indígenas originarias y que fue abandonado en 1541 trasladándose su población al Paraguay y el segundo constituyó el inicio que llevó a la conformación de la ciudad actual.

La CABA tiene una superficie de $202 \mathrm{~km}^{2}$ con 2.890 .151 habitantes (46\% varones y $54 \%$ mujeres) y es la ciudad central de una aglomeración urbana Gran Buenos Aires, de 12.806.866 habitantes (INDEC, 2010). Los grandes grupos de edad indican que su población está compuesta por un 16,35\% entre 0 y 14 años, $67,25 \%$ de 15 a 64 años y 16,40\% de 65 años y más. Su localización en el espacio absoluto es $34^{\circ} 36^{\prime} 37^{\prime \prime}$ Sur y $58^{\circ} 23^{\prime} 22^{\prime \prime}$ Oeste, considerando este punto como kilómetro cero de la red de caminos en Argentina ubicado en la Plaza Moreno frente al edificio del Congreso Nacional.

Su división político-administrativa interna son 15 comunas que agrupan los 48 barrios tradicionales en que sus habitantes conocen los diferentes sectores 
de la ciudad. El Cuadro $N^{\circ} 1$ muestra esta correspondencia y la Figura $\mathrm{N}^{\circ} 1$ mapas de situación relativa en diferentes contextos y escalas.

\section{Figura $\mathrm{N}^{\circ}$ 1. Ciudad Autónoma de Buenos Aires. Situación relativa.}

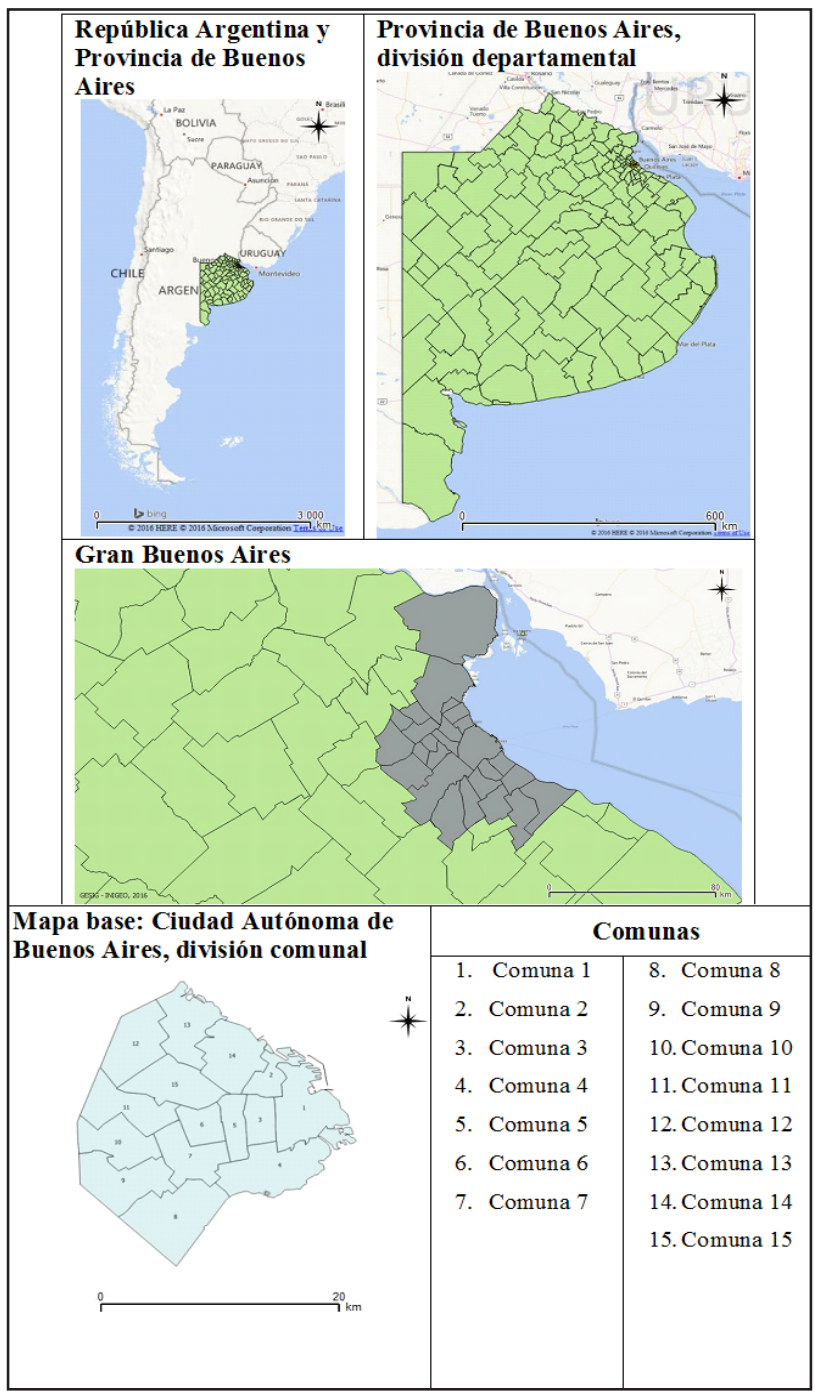

Fuente: Elaboración propia. 


\section{Cuadro $N^{\circ}$ 1. Unidades espaciales de la Ciudad Autónoma de Buenos Aires}

\begin{tabular}{|c|l|}
\hline Comuna & Barrios \\
\hline 1 & $\begin{array}{l}\text { Constitución, Montserrat, Puerto Madero, Retiro, San Nicolás y } \\
\text { San Telmo. }\end{array}$ \\
\hline 2 & Recoleta \\
\hline 3 & Balbanera y San Cristobal \\
\hline 4 & Barracas, La Boca, Nueva Pompeya y Parque Patricios \\
\hline 5 & Almagro y Boedo \\
\hline 6 & Caballito \\
\hline 7 & Flores y Parque Chacabuco \\
\hline 8 & Villa Lugano, Villa Riachuelo y Villa Soldati \\
\hline 9 & Liniers, Matadero y Parque Chacabuco \\
\hline 10 & $\begin{array}{l}\text { Floresta, Monte Castro, Villa del Parque, Villa General Mitre, } \\
\text { Villa Luro y Villa Real }\end{array}$ \\
\hline 11 & $\begin{array}{l}\text { Villa del Parque, Villa del Parque, Villa Devoto y Villa General } \\
\text { Mitre }\end{array}$ \\
\hline 12 & Coghland, Saavedra, Villa Pueyrredón y Villa Urquiza \\
\hline 13 & Belgrano, Colegiales y Nuñez \\
\hline 14 & Palermo \\
\hline 15 & $\begin{array}{l}\text { Agronomía, Chacarita, La Paternal, Parque Chas, Villa Crespo y } \\
\text { Villa Ortuzar }\end{array}$ \\
\hline
\end{tabular}

\section{El mapa social de la Ciudad Autónoma de Buenos Aires}

Consideramos como antecedentes el análisis del mapa social del Gran Buenos Aires 2001, realizado a partir de la aplicación de técnicas de análisis multivariado (Buzai y Marcos, 2012) y, la actualización 2010 realizada a partir de la distribución espacial del Índice de Calidad de Vida (ICV) (Baxendale et al., 2016). En ambos casos, la estructura espacial resultante fue interpretada mediante modelos urbanos propuestos para las grandes ciudades de América Latina (Buzai, 2014, 2016). 
El ICV es un índice sintético obtenido mediante la combinación de índices parciales para las variables consideradas. Su construcción fue realizada por Velázquez (2016) y contempla 2 dimensiones compuestas de 5 grandes temáticas: Dimensión socioeconómica (educación, salud y vivienda) y (2) Dimensión ambiental (problemas ambientales y recursos recreativos).

La estandarización de las 29 variables para la formación de índices parciales (macro-variables) se construye generando puntajes omega, tanto para variables de beneficio $[\mathrm{vb}=(\mathrm{x}-\mathrm{m}) /(\mathrm{M}-\mathrm{m})]$ como de costo invertidas $[\mathrm{vc}=(\mathrm{M}-\mathrm{x}) /(\mathrm{M}-\mathrm{m})] \mathrm{a}$ ser combinadas en el ICV, resultado único de ranking que adquiere cada unidad espacial. ${ }^{3}$ Del trabajo de Baxendale et al. (2016) fueron tomados los resultados del ICV de cada Comuna de la CABA y su distribución espacial se presenta en la Figura $\mathrm{N}^{\circ} 2$.

\section{Figura $\mathrm{N}^{\circ}$ 2. Distribución espacial del ICV como indicador del mapa social de la Ciudad Autónoma de Buenos Aires}

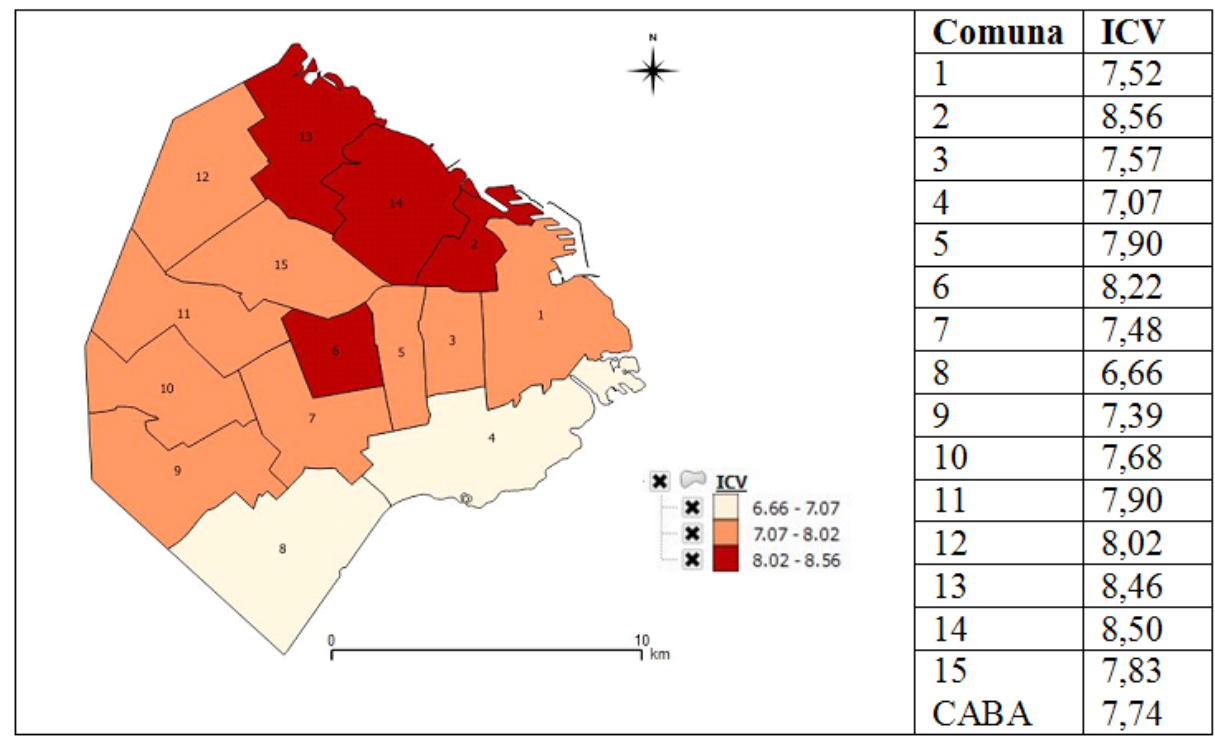

Fuente: Elaboración propia. Método de clasificación de cortes naturales, 3 categorías.

\footnotetext{
${ }^{3}$ vc es la variable de costo, vb es la variable de beneficio, $\mathrm{x}$ es el valor de la variable, $\mathrm{M}$ es el dato mayor de la variable y $\mathrm{m}$ es el dato menor de la variable. Los resultados de cada fórmula multiplicados por la decena llevará los valores entre 0 (para el dato menor) y 10 (para el dato mayor).
} 
El mapa social de la CABA realizado a través del mapeo del ICV presenta su tradicional división socioespacial interna. Las clases sociales altas con mayores valores en el ICV se encuentran principalmente en el norte de la ciudad, las clases sociales medias en el centro y las clases sociales bajas con menores valores en el ICV en el sur. Las condiciones económicas, en el máximo nivel educativo alcanzado y en las condiciones de atención médica, van empeorando de norte a sur.

La realización del mapa social resulta la base para un análisis exploratorio en la búsqueda de asociaciones espaciales con el comportamiento electoral, es decir, las relaciones espaciales entre características fundamentales de la población y los hechos políticos. Esta aproximación permitirá comprobar aspectos subyacentes que llevan a la preferencia por determinados partidos políticos.

\section{ANÁLISIS ESPACIAL DE LA ELECCIÓN A JEFE DE GOBIERNO DE LA CIUDAD AUTÓNOMA DE BUENOS AIRES}

\section{La elección}

El día 26 de abril de 2015 se realizaron las Elecciones Abiertas, Primarias y Obligatorias (PASO), una elección primaria que funciona como definición interna de cada partido político a fin de definir el candidato que participará de la elección general. Pasar a la siguiente instancia se logra si se ha obtenido como mínimo el 1,5\% de los votos y se es ganador en su espacio político. Son Abiertas porque participan todos los ciudadanos habilitados independientemente que se encuentren o no afiliados a algún partido político, Primarias porque se elegirán inicialmente las candidaturas oficiales, no los cargos, y Obligatorias para toda la ciudadanía comprendida entre los 18 y 70 años de edad, siendo optativo para edades superiores.

El día 5 de Julio de 2015 se realizó la elección de Jefe de Gobierno para la CABA luego de haberse definido las PASO, a partir de las cuales fueron habilitados aquellos candidatos ganadores en sus espacios políticos y cuyos partidos hayan obtenido un 1,5\% o más de los votos. El Cuadro $\mathrm{N}^{\circ} 2$ presenta los que obtuvieron esas cifras.

\section{Partidos políticos con intención mayoritaria de votos}

Propuesta Republicana (PRO)

Tiene existencia desde el 2008, aunque su reconocimiento comienza tres años antes con la creación de Compromiso para el Cambio y su alianza con 
varios sectores políticos que apoyan la ideología del liberalismo económico. Este espacio político tiene su principal presencia en la CABA y gana las elecciones municipales de los años 2007 y 2011. De esta manera lleva al cargo de Jefe de Gobierno a Mauricio Macri quien, después de dos mandatos, se define por la carrera presidencial para las elecciones 2015.

Para las elecciones 2015 en la CABA, el PRO presenta dos precandidatos para enfrentarse en las PASO: Horacio Rodríguez Larreta y Gabriela Michetti. Como candidato presidencial se apoya a Mauricio Macri.

\section{Frente para la Victoria (FPV)}

Espacio político que resulta de una alianza realizada en el año 2003 para apoyar la candidatura presidencial de Néstor Kirchner. El Partido Justicialista es el principal grupo político que la integra y se apoyan en la ideología del peronismo combativo.

Este espacio político tiene su principal presencia a nivel nacional y gana las elecciones nacionales de los años 2003, 2007 y 2011. La primera lleva al cargo de Presidente de la Nación a Néstor Kirchner y la segunda y tercera a su esposa Cristina Fernández de Kirchner.

Para las elecciones 2015 en la CABA, el FPV presenta siete precandidatos para enfrentarse en las PASO: Mariano Recalde, Gabriela Cerutti, Anibal Ibarra, Carlos Heller, Gustavo López, Víctor Ramos y Calos Oviedo. Como candidato presidencial se apoya a Daniel Scioli, el actual gobernador de la Provincia de Buenos Aires y garante de continuación del proyecto político desarrollado durante más de una década a nivel nacional.

\section{Energía Ciudadana Organizada (ECO)}

Es un espacio político que surge del Frente Amplio (UNEN) para intervenir en las elecciones municipales de la CABA en las elecciones 2015. Se apoya en una coalición política de centro-izquierda entre los que se destaca el tradicional partido de la Unión Cívica Radical (UCR).

Para las elecciones 2015 en la CABA, el ECO presenta dos precandidatos para enfrentarse en las PASO: Martín Lousteau y Graciela Ocaña. Como candidatos presidenciales del Frente Ampio (UNEN) se presentarán en las primarias junto al PRO en un nuevo espacio denominado "Cambiemos". La interna se dirime entre Mauricio Macri (PRO) y Ernesto Sanz (Frente Ampio UNEN).

En las PASO se presentaron 16 agrupaciones políticas y cinco han conseguido los porcentajes de votos que les permiten estar habilitados para participar de la 
elección general. Los votos obtenidos y sus porcentajes en el padrón electoral han sido los siguientes: PRO (882.421 votos, $48,06 \%$ ), ECO (413.465 votos, $22,48 \%$ ), FPV (384.282 votos, 18,93), FIT (Frente de Izquierda de los trabajadores) (41.719 votos, 2,26\%) y AyL (Autodeterminación y Libertad) (37.885 votos, 2,05\%).

\section{Elección a Jefe de Gobierno de la CABA}

El día 5 de Julio de 2015 se realizó la elección general de Jefe de Gobierno para la CABA luego de haberse definido las PASO a partir de las cuales fueron habilitados aquellos candidatos ganadores en sus espacios políticos y cuyos partidos hayan obtenido un 1,5\% o más de los votos. El Cuadro $\mathrm{N}^{\circ} 2$ presenta las cifras (ver Cuadro $\mathrm{N}^{\circ} 2$ ). Los resultados definen la realización de un ballotage o segunda vuelta entre los dos partidos con mayor cantidad de votos (La Nación, 2015a), ya que ninguno de ellos logró superar el 50\% de los votos emitidos, condición exigida por la ley electoral de la CABA para ser triunfador directo en las elecciones generales.

\section{Cuadro $\mathrm{N}^{\circ}$ 2. Ciudad Autónoma de Buenos Aires. Resultado de las elecciones generales}

\begin{tabular}{|l|l|l|l|}
\hline Partido & \multicolumn{2}{l}{ Fórmula } & 818.964 \\
\hline PRO & $\begin{array}{l}\text { Horacio Rodríguez Larreta - } \\
\text { Diego Santilli }\end{array}$ & 45,50 \\
\hline ECO & $\begin{array}{l}\text { Martín Lousteau - Fernando } \\
\text { Sánchez }\end{array}$ & 458.902 & 25,50 \\
\hline FPV & $\begin{array}{l}\text { Mariano Recalde - Leandro } \\
\text { Santoro }\end{array}$ & 394.541 & 21,90 \\
\hline AyL & Luis Zamora - Sergio Sallustio & 71.223 & 4,00 \\
\hline FIT & Myriam Bergman - José Castillo & 56.038 & 3,10 \\
\hline & Votos en blanco y nulos & 33.870 & 1,90 \\
\hline
\end{tabular}

\section{Distribuciones espaciales de la elección general a Jefe de Gobierno de la CABA}

La distribución espacial de los votos de los partidos políticos mayoritarios se presenta en la Figura $\mathrm{N}^{\circ} 3$. Cabe ser destacado, que el PRO constituye la continuidad del gobierno de la CABA y el FPV es el partido político del gobierno nacional, que nunca pudo ganar en la ciudad. En las elecciones 2007 y 2011 hubo ballotage, pero en ambos casos el candidato Daniel Filmus no pudo superar los votos obtenidos por Mauricio Macri, actual candidato presidencial en las elecciones nacionales 2015. 
Figura $\mathrm{N}^{\circ}$ 3. Distribución espacial de los votos en la elección general a Jefe de Gobierno de la CABA

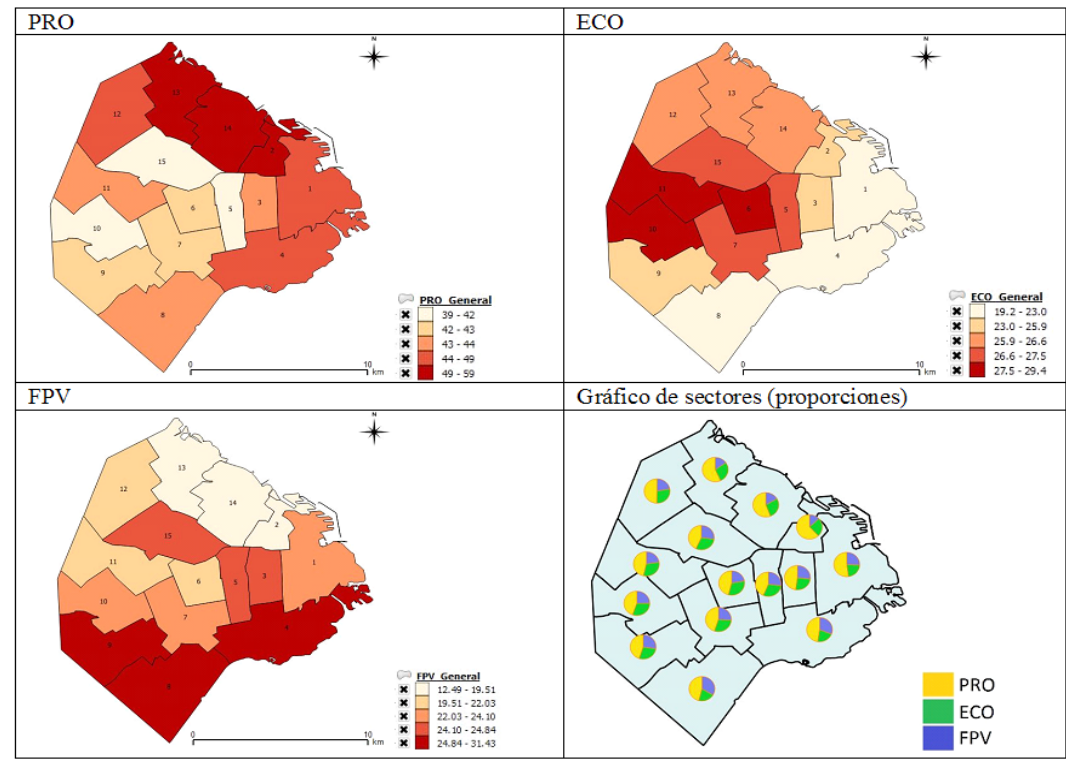

Fuente: Elaboración propia

A continuación se explora la relación entre la distribución espacial del ICV y el voto realizado a los principales partidos políticos (Figura $\mathrm{N}^{\circ} 4$ ).

Figura $N^{\circ}$ 4. Gráficos de dispersión, ICV y votos

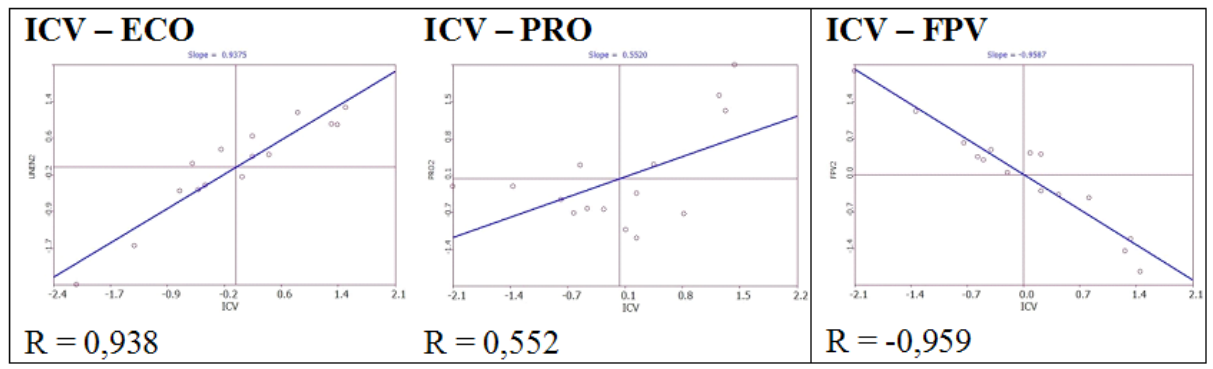

Fuente: Elaboración propia 
Los resultados indican que son dos los partidos que se relacionan de manera directa con el ICV. Indica que a mayor ICV hay mayor cantidad de votos para ECO y PRO, y de manera inversa, las unidades espaciales con menor ICV presentan mayor cantidad de votos para el FPV. Si se considera una situación causal, a través del coeficiente $\mathrm{r}^{2}$ se podría decir que en los extremos el ICV explica el 92\% de los votos obtenidos por el FPV y el $88 \%$ de los obtenidos por el ECO. Según Gervasoni (2015), normalmente se asume que el peronismo gana en aquellas zonas en las que hay pobreza y su trabajo intenta mostrar de qué manera esta situación está cambiando a nivel nacional, sin embargo, el presente trabajo comprueba con fuerza esa afirmación.

A continuación, avanzamos focalizando nuestra atención a la asociación espacial entre los votos y la dimensión educativa a través de la variable Máximo Nivel Educativo Alcanzado, siendo el primario completo una variable de costo y el secundario y universitario completos variables de beneficio. El análisis será realizado a través de los siguientes gráficos de dispersión y los correspondientes coeficientes de correlación (ver Figura $\mathrm{N}^{\circ} 5$ ). El análisis de la Figura $\mathrm{N}^{\circ} 5$ por columnas presenta la relación del voto para cada uno de los partidos políticos y las tres variables educativas en valores absolutos estandarizados ambas variables en puntajes $z$.

El PRO y el ECO muestran comportamientos similares con puntajes negativos hacia los máximos niveles educativos alcanzados primario y secundario completo, y positivo hacia el terciario completo. El PRO es el que tiene una relación más intensa. El FPV presenta un comportamiento inverso con puntajes positivos hacia las dos primeras variables y negativo al terciario-universitario completo. El valor máximo positivo lo presenta con el primario completo y el máximo negativo con el terciario universitario.

En síntesis, de ambos análisis exploratorios surge que en la CABA, el PRO y el ECO obtienen principalmente sus electores de las áreas con mayor ICV y nivel educativo, y que el FPV, de manera inversa, de las áreas con menor ICV y menor nivel educativo. De la misma forma es posible pensar el funcionamiento de toda variable de costo y beneficio, comprobando, al menos en la CABA, la hipótesis que Gervasoni (2015) pone en duda a nivel nacional. En este sentido, el mapa social adquiere un peso muy importante en el resultado final de la elección. 
Figura $\mathrm{N}^{\circ}$ 5. Relación entre el voto a Jefe de Gobierno de la CABA y el máximo nivel educativo alcanzado

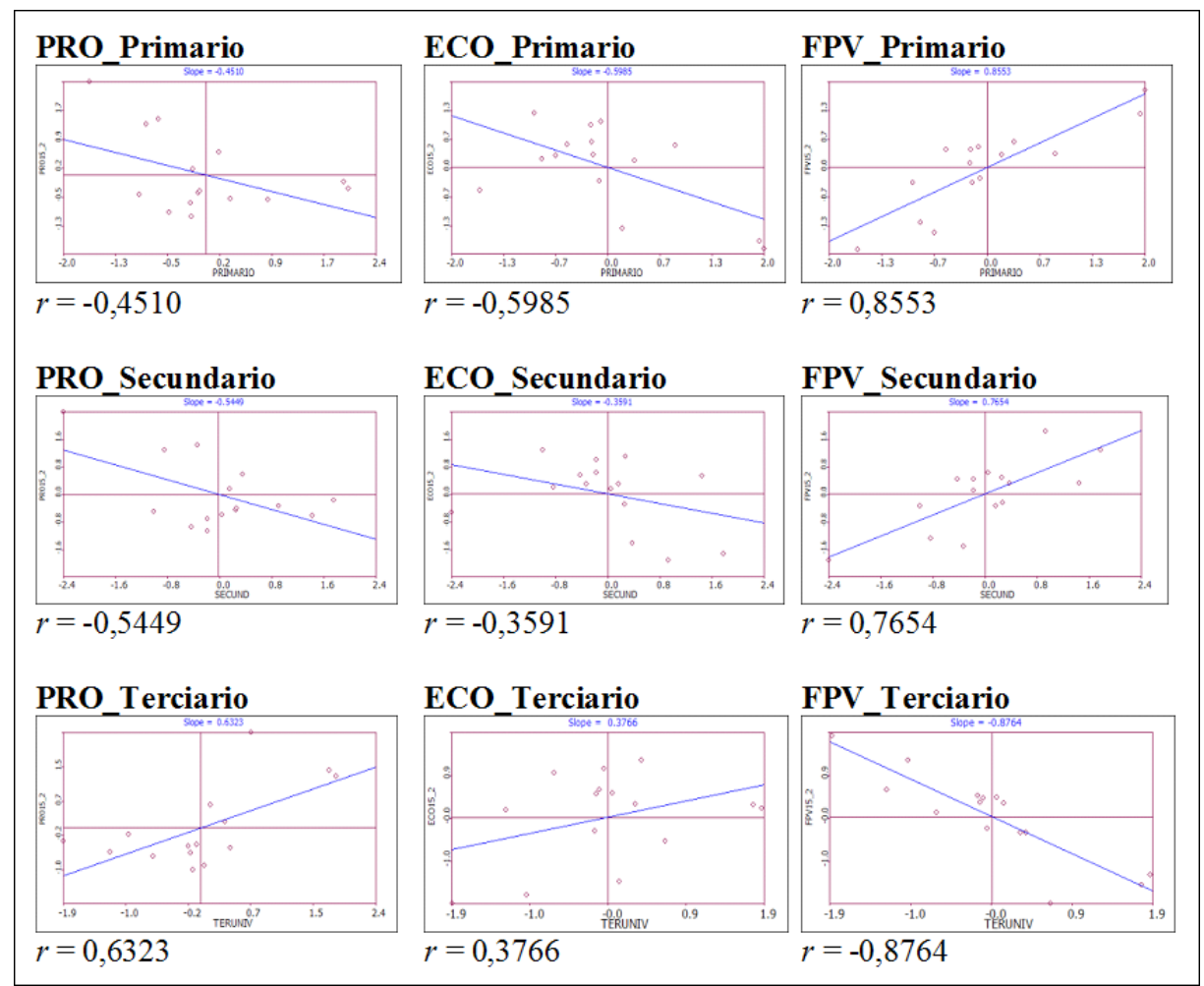

Fuente: Elaboración propia

Resultado y distribución espacial de los votos en el 'ballotage' a Jefe de Gobierno de la CABA

El día 19 de Julio de 2015 se realizó la segunda vuelta entre los dos partidos ganadores y fue definido el nuevo Jefe de Gobierno de la CABA en base a los resultados que presenta la Cuadro $\mathrm{N}^{\circ} 3$. 
Cuadro $\mathrm{N}^{\circ}$ 3. Ciudad Autónoma de Buenos Aires. Resultado del ballotage a Jefe de Gobierno

\begin{tabular}{|l|l|l|l|}
\hline Partido & Fórmula & 860.802 & 51,06 \\
\hline PRO & $\begin{array}{l}\text { Horacio Rodríguez Larreta - Diego } \\
\text { Santilli }\end{array}$ & 806.057 & 48,04 \\
\hline ECO & Martín Lousteau - Fernando Sánchez & 104.445 & 5,80 \\
\hline & Votos en blanco y nulos & 8 . \\
\hline
\end{tabular}

Comparando los resultados presentados en el Cuadro $\mathrm{N}^{\circ} 2$ con los del Cuadro $\mathrm{N}^{\circ} 3$, se pone en evidencia una directa transferencia de votos desde el FPV para el ECO, inclusive algún analista, previo al ballotage, había pronosticado que esto los llevaría a ganar las elecciones (Ramos, 2015), cosa que no sucedió por tres puntos (La Nación, 2005b), aunque fue vencedor en varias comunas (La Nación, 2015c). De esta manera, el partido político gobernante en la CABA mantenía el distrito bajo su administración por tercer período consecutivo, ahora en la fórmula Larreta-Santilli por los próximos cuatro años (ver Figura $\mathrm{N}^{\circ} 6$ ).

Figura $\mathrm{N}^{\circ}$ 6. Distribución espacial de los votos en el ballotage a Jefe de Gobierno de la CABA

PRO

Fuente: Elaboración propia 


\section{ANÁLISIS ESPACIAL DE LA ELECCIÓN A PRESIDENTE DE} LA REPÚBLICA ARGENTINA

\section{La elección}

El 9 de agosto de 2015 se realizaron las PASO teniendo las mismas características que las ya enunciadas en un punto anterior. A partir de sus resultados se determinan los partidos políticos y candidatos habilitados para la elección general. El 25 de octubre de 2015 se realiza la elección general para la presidencia de la República Argentina y, en el caso de que ninguno de los candidatos obtuviera más del $45 \%$ de los votos o más del $40 \%$ de los votos con una distancia de 10 puntos porcentuales al segundo, sería realizada una segunda vuelta o ballotage el 22 de noviembre del mismo año.

\section{Partidos políticos con intención mayoritaria de votos}

\section{Frente para la Victoria (FPV)}

Es el partido político en ejercicio del poder a nivel nacional. Al quedar trunca la idea de realizar una reforma constitucional que habilite a la presidente Cristina Fernández de Kirchner a presentarse a elecciones para un tercer mandato, ella personalmente elige a Daniel Scioli, actual gobernador de la Provincia de Buenos Aires, como postulante que garantiza la continuidad del proyecto del gobierno nacional y al cual se le brinda todo el apoyo partidario. A partir de esta elección del candidato del partido oficial, realizada de forma verticalista y direccionada, queda imposibilitada una elección interna entre diferentes postulantes (Obarrio, 2015) y el FPV se presenta a las PASO con una única posibilidad. Daniel Scioli unificará los votantes que tienen convicción por el candidato y los que no la tienen pero apoyan el proyecto político.

\section{Cambiemos}

Es un espacio político formado en 2015 a partir de la coalición de tres partidos políticos: Propuesta Republicana (PRO), Unión Cívica Radical (UCR) y Coalición Cívica para la Afirmación de una República Igualitaria (CC-ARI). Cambiemos presenta tres candidatos presidenciables para las PASO: Mauricio Macri (PRO), Ernesto Sanz (UCR) y Elisa Carrio (CC-ARI).

\section{Unidos por una Nueva Alternativa (UNA)}

Es un espacio político formado en 2015 a partir de una coalición surgida del 
peronismo tradicional con ideología de centro-derecha a partir de una separación y oposición con el FPV. Los candidatos que UNA presenta para las PASO son Sergio Massa (Diputado Nacional por la Provincia de Buenos Aires) y José Manuel de la Sota (Gobernador de la Provincia de Córdoba). En las PASO se presentaron 11 agrupaciones políticas y seis han conseguido los porcentajes de votos que les permiten estar habilitados para participar de la elección general.

Los votos obtenidos y sus porcentajes en el padrón electoral han sido los siguientes: FPV (8.720.573 votos, 38,67\%), PRO (6.791.278 votos, 30,12\%), UNA (Unidos por una Nueva Alternativa) (4.639.405 votos, 20,57\%), GEN (Generación para un Encuentro Nacional) (781.472 votos, 3,47\%), FIT (Frente de Izquierda de los Trabajadores) (375.874 votos, 3,25\%) y ACF (Alianza Compromiso Federal) (472.341 votos, $2,09 \%)$.

\section{Elección a Presidente de la República Argentina}

El día 25 de Octubre de 2015 se realizó la elección a la Presidencia de la República Argentina, luego de haberse definido las PASO a partir de las cuales fueron habilitados aquellos candidatos ganadores en sus espacios políticos y cuyos partidos hayan obtenido un $1,5 \%$ o más de los votos. El Cuadro $\mathrm{N}^{\circ} 4$ presenta las cifras.

\section{Cuadro $N^{\circ}$ 4. Resultado de las elecciones presidenciales}

\begin{tabular}{|l|l|l|l|}
\hline Partido & Fórmula & 9.338 .449 & 37,08 \\
\hline FPV & Daniel Scioli-Carlos Zannini & 8.601 .063 & 34,15 \\
\hline PRO & Mauricio Macri-Gabriela Michetti & 5.386 .965 & 21,39 \\
\hline UNA & Sergio Massa-Gustavo Sáenz & 812.530 & 3,23 \\
\hline FIT & Nicolás del Caño-Myriam Bregman & 632.551 & 2,51 \\
\hline GEN & Margarita Stolbizer-Miguel Olaviaga & 412.577 & 1,64 \\
\hline ACF & Adolfo Rodríguez Saá-Liliana Negre & 637.639 & 2,46 \\
\hline & Votos en blanco y nulos & & \multicolumn{2}{l}{. } \\
\hline
\end{tabular}

Los resultados definen la realización de un ballotage o segunda vuelta entre los dos partidos con mayor cantidad de votos, ya que ninguno de ellos logró el porcentaje necesario para ser triunfador directo en las elecciones generales. 
Distribuciones espaciales de la elección general a Presidente de la República Argentina en la CABA

La distribución espacial de los votos de los partidos políticos mayoritarios se presenta en la Figura $\mathrm{N}^{\circ} 7$ Cabe ser destacado que el PRO constituye la continuidad del gobierno de la CABA y el FPV es el partido político del gobierno nacional, que nuca pudo ganar en la ciudad. En las elecciones 2007 y 2011 hubo ballotage, pero en ambos casos el candidato Daniel Filmus no pudo superar los votos obtenidos por Mauricio Macri, actual candidato presidencial en las elecciones nacionales 2015 .

\section{Figura $\mathrm{N}^{\circ} 7$. Distribución espacial de los votos en la elección general a Presidente de la República Argentina}

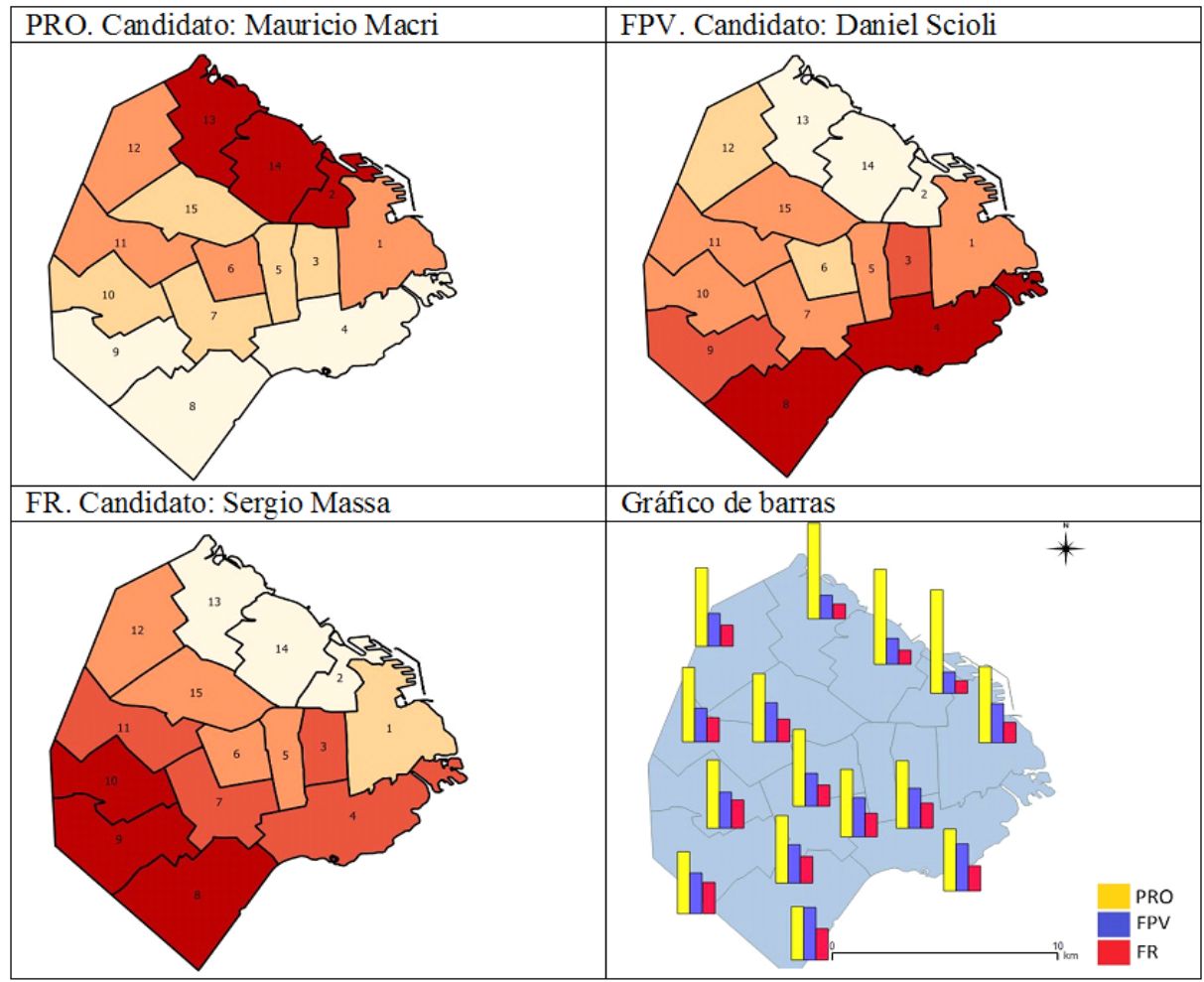

Fuente: Elaboración propia. Método de cortes naturales 5 intervalos 
Los resultados indican que solamente un partido político se relaciona de manera directa con el ICV, a mayor ICV hay mayor cantidad de votos para el PRO, y de manera inversa las unidades espaciales con menor ICV presentan mayor cantidad de votos para el FPV y el FR, ambos de extracción justicialista (Peronismo). Si se considera una situación causal, a través del coeficiente $r^{2}$ se podría decir que en los extremos el ICV explica el 88\% de los votos obtenidos por el PRO y el 92\% de los obtenidos por el FPV. Estos datos refuerzan aún más la consideración de que el peronismo obtiene sus votos de las zonas socialmente desfavorecidas (ver Figura $\mathrm{N}^{\circ} 8$ ).

\section{Figura $\mathrm{N}^{\circ}$ 8. Gráficos de dispersión entre ICV y votos}

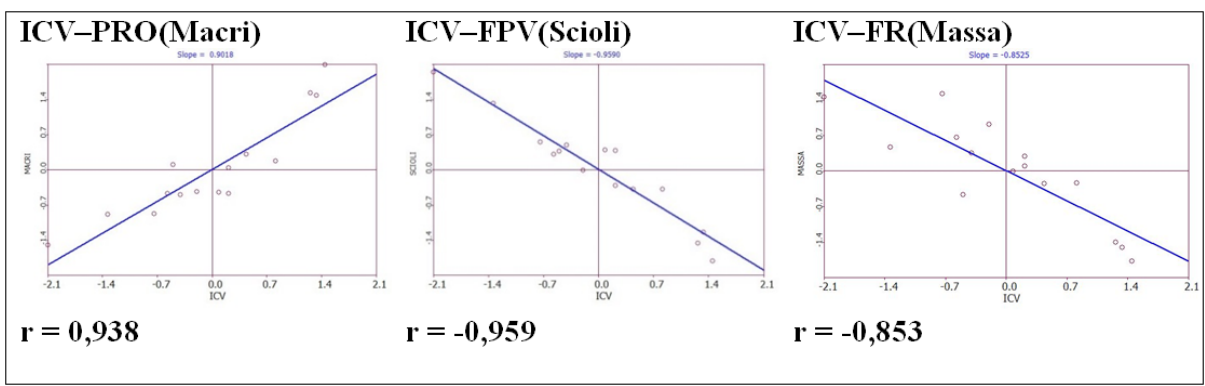

Fuente: Elaboración propia.

A continuación avanzamos focalizando nuestra atención a la asociación espacial entre los votos y la dimensión educativa a través de la variable Máximo Nivel Educativo Alcanzado, variables con las características ya verificadas. El análisis será realizado a través de los siguientes gráficos de dispersión y los correspondientes coeficientes de correlación. 


\section{Figura $\mathrm{N}^{\circ}$ 9. Relación entre el voto a Presidente de la República Argentina y el} máximo nivel educativo alcanzado

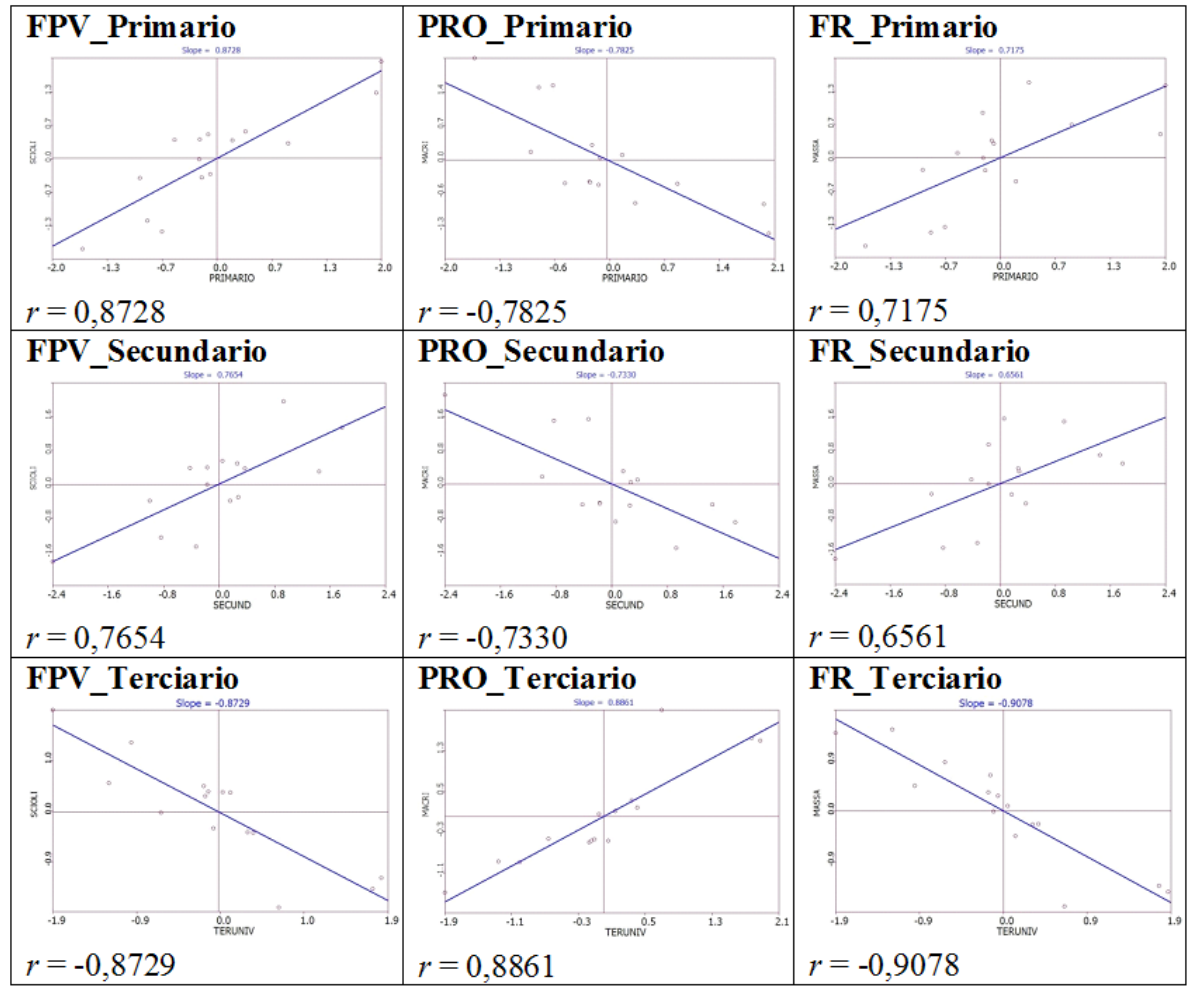

Fuente: Elaboración propia.

El análisis de la Figura $\mathrm{N}^{\circ} 8$ por columnas presenta la relación del voto para cada uno de los partidos políticos y las tres variables educativas.

El FPV y el FR muestran comportamientos similares con puntajes positivos hacia los máximos niveles educativos alcanzados primario y secundario completo, y negativo hacia el terciario completo. El PRO presenta un comportamiento inverso con puntajes positivos con el máximo nivel educativo alcanzado terciario-universitario completo.

En síntesis, de ambos análisis exploratorios surge que en la CABA, el FPV y el FR obtienen principalmente sus electores de las áreas con bajo y medio ICV y nivel 
educativo, y que el PRO, de manera inversa, de las áreas con mayor ICV y mayor nivel educativo alcanzado.

Resultado y distribución espacial de los votos en el ballotage a Presidente de la República Argentina

El día 22 de Noviembre de 2015 se realizó la segunda vuelta entre los dos partidos ganadores y fue definido el nuevo Presidente de la República Argentina en base a los resultados que presenta la Cuadro $\mathrm{N}^{\circ} 5$.

Cuadro $\mathrm{N}^{\circ}$ 5. Ciudad Autónoma de Buenos Aires. Resultado del ballotage a Presidente de la Nación

\begin{tabular}{|l|l|l|l|}
\hline Partido & Fórmula & Votos & Votos \% \\
\hline PRO & Mauricio Macri - Gabriela Michetti & 12.997 .937 & 51,34 \\
\hline FPV & Daniel Scioli - Carlos Zaninni & 12.317 .330 & 48,66 \\
\hline & Votos en blanco y nulos & 637.639 & $2,46 \%)$ \\
\hline
\end{tabular}

Figura $\mathrm{N}^{\circ}$ 10. Distribución espacial de los votos en el ballotage a Presidente de la República Argentina

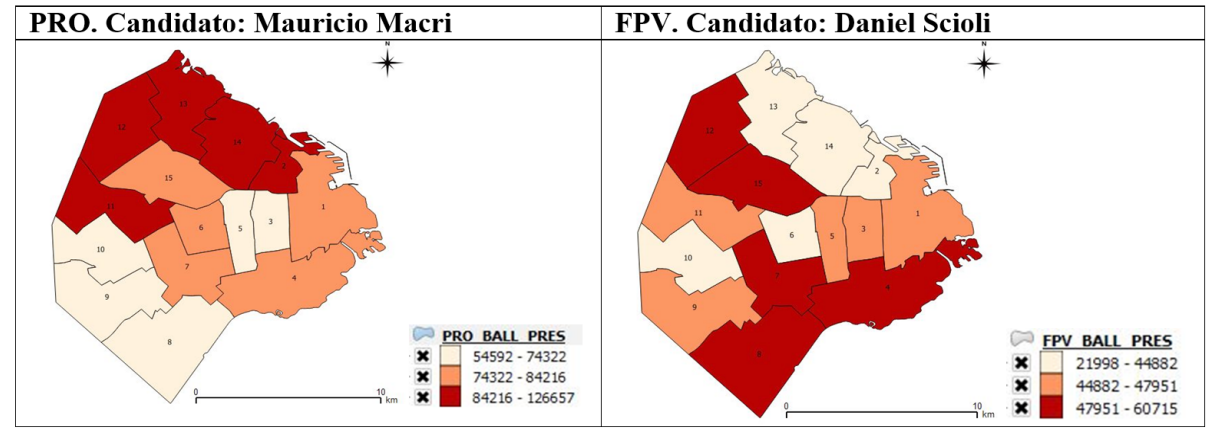

Fuente: Elaboración propia.

Comparando los resultados presentados en el Cuadro $\mathrm{N}^{\circ} 4$ con los del Cuadro $\mathrm{N}^{\circ} 5$ surge que no hubo transferencia directa de votos de un partido a otro y que 
los votos de los partidos perdedores se reparten entre los candidatos del ballotage con preferencia hacia el PRO que suma 17,19 puntos porcentuales frente al FPV que lo hace en 11,58, de acuerdo a los gráficos de la Figura $N^{\circ} 8$ esta transferencia realizada desde los sectores medios. De esta manera el partido político gobernante en la República Argentina pierde el ballotage y se proclama a la fórmula integrada por Mauricio Macri y Gabriela Michetti para asumir los cargos de Presidente y Vicepresidente de la República Argentina a partir del 10 de diciembre de 2015 por los próximos cuatro años.

\section{CONSIDERACIONES FINALES}

En el año 2015 la CABA tuvo un rol importante en la Geografía Electoral de la República Argentina. En la ciudad capital de la República Argentina el PRO tuvo un gran protagonismo siendo el ganador de las elecciones a Jefe de Gobierno de la CABA y a Presidente de la República Argentina. En el primer caso correspondiendo a un tercer mandato consecutivo y en el segundo desplazando al FPV de su posible continuidad.

De acuerdo a los resultados obtenidos, el PRO obtuvo mayor cantidad de votos en la totalidad de comunas de la CABA, pero sus mayores correlaciones con el mapa social estuvieron dadas con la población que ostenta el mayor ICV y máximo nivel educativo alcanzado. En líneas generales las clases sociales media-altas y altas, aquellas que tienen mejores condiciones de vida apoyan una ideología orientada al liberalismo económico.

El PRO fue un claro ganador en la CABA en la elección a Jefe de Gobierno en las PASO y en la elección general, sin embargo en el ballotage la transferencia de votos desde el FPV al ECO hizo que finalmente ganara por una pequeña diferencia cercana a 2 puntos en lo que resultó ser una competencia contra dos partidos. En este caso fue central el efecto de grupo a partir de las condiciones que propiciaron el voto en contra del PRO.

Algo diferente resultó ser la elección a Presidente de la República Argentina. Tanto en las PASO como en la elección general fue ganador el FPV y segundo el PRO, aunque por una diferencia de votos que no permitió resolver el resultado final en primera vuelta. El tercer partido, PR repartió sus votos entre ambos partidos y el PRO resultó ganador. El efecto vecindario incrementó los resultados en todos los sectores de la CABA principalmente la mayor incidencia de la clase media hacia el PRO. 
En ambas instancias (Jefe de Gobierno, Presidencia de la República) se determinan claras asociaciones espaciales entre la distribución de los votos, el mapa social y las condiciones del máximo nivel educativo alcanzado de la población. Las zonas con medio-altos y altos ICV y máximos niveles educativos alcanzados resultaron fundamentales en la definición final de los resultados. El efecto de grupo se produjo ante la transferencia directa de votos de un partido a otro, el efecto de vecindario tuvo su mayor relación directa con el mapa social de la ciudad.

La Geografía Electoral puede descubrir comportamientos significativos en la elección ideológica de las personas en cuanto a su contexto socio-espacial. La CABA ha sido un gran ejemplo a partir de dos niveles electorales en procesos cíclicos que se producen entre momentos de estabilidad y crisis, como cambios paradigmáticos.

\section{BIBLIOGRAFÍA}

Baxendale, C. A., Buzai, G. D. \& Morina, J. O. (2016). La Región Metropolitana de Buenos Aires. En Velázquez, G. A. 2016. Geografía y Calidad de Vida en Argentina. Análisis regional y departamental 2010. (pp. 261-281). Tandil: Universidad Nacional del Centro de la Provincia de Buenos Aires.

Bosque Sendra, J. (1980). Influencias espaciales en el comportamiento electo $\neg$ ral: el efecto de amigos y vecinos (votaciones al Senado, ciudad de Granada, 15 de junio de 1977). Revista Internacional de Sociología. 36, 577594.

Bosque Sendra, J. (1981a). Procesos de contagio espacial en el comportamiento electoral de la población española (1977 1979). Anales de Geografía de la Universidad Complutense. $1,97115$.

Bosque Sendra, J. (1981b). Las campañas de mítines electorales en Granada (15 J 77 y 1 M 79). Revista de Estudios Políticos. 22, 237250.

Bosque Sendra, J. (1982). Modelos ecológicos del comportamiento electoral en España (1977 79). Estudios geográficos. 166, 3359.

Bosque Sendra, J. (1988). Geografía Electoral. Madrid: Síntesis. Busteed, M. A. (1975). Geography and voting behaviour. London: Oxford University Press.

Buzai, G. D. (2014). Mapas Sociales Urbanos. Buenos Aires: Lugar Editorial.

Buzai, G. D. (2016). Urban Models in the study of Latin American cities. Inssbrucker Geographische Studien. 40, 91-108.

Buzai, G. D., \& Marcos, M. (2012) The Social Map of Greater Buenos Aires as Empirical Evidence of Urban Models. Journal of Latin American Geography. 11 (1), 67-78.

Cox, K. R. (1969). The voting decision in spatial context. Progress in Geography. 1, 40-53. 
Cox, K. R. (1971). The spatial component of urban voting response surfaces. Economic Geography. 47, 27-35.

Cox, K. R. (1998). Classics in Human Geography revisited: The voting decision in spatial context. Progress in human Geography. 22 (3), 407-413.

Escandon, C. \& López Levi, L. (2004.) Sistemas de información geográfica y redistritación electoral: su impacto en México. GeoCrítica / Scripta Nova. Revista electrónica de geografía y ciencias sociales. Barcelona: Universidad de Barcelona, VIII, 170(63). Recolectado de: http://www.ub.es/geocrit/sn/sn-170-63.htm.

Gervasoni, C. (2015). Cuatro años para reconfigurar la geografía electoral. Diario Alfil.: Recolectado de: http://www.diarioalfil.com.ar/pagina/2015/11/26/cuatro-anos-parareconfigurar-la geografia-electoral/.

Gombin, J. (2014). Contextualiser sans faire de l'espace un facteur autonome. La modélisation multiniveau comme lieu de rencontre entre sociologie et géographie électorales, L'Espace Politique, 2. Recolectado de: http://espacepolitique.revues.org/3066

Gould, P. (2000). Pensar como un geógrafo. Una exploración en la Geografía Moderna. Scripta Nova. Revista Electrónica de Geografía y Ciencias Sociales. 78. Recolectado de: http:// www.ub.edu/geocrit/sn-78.htm.

INDEC. (2010). Censo Nacional de Población, Hogares y Viviendas 2010. Buenos Aires: Instituto Nacional de Estadística y Censos.

Johnston, R.; Pattie, Ch. (2012.) Kevin Cox and electoral geography. En Jonas, A. \& Wood, A. (Eds.) Territory, the state and urban politics: a critical appreciation of the selected writings of Kevin R. Cox. (pp. 23-44). Ashgate: Burlington VT.

Jones, K., Johnston, R., \& Pattie, Ch. (1992). People, Places, and Regions: Exploring the Use of Multi-Level Modeling in the Analysis of Electoral Data. British Journal of Political Science. 22 (03), 343-380.

La Nación (19 de julio de 2015b). Horacio Rodriguez Larreta ganó el ballotage por una ajustada diferencia. La Nación. Recolectado de: http://www.lanacion.com.ar/1811801.

La Nación (19 de julio de 2015c). Lousteau se impuso en 9 de las 15 comunas: mirá el mapa interactivo de La Nación. La Nación. Recolectado de: http://www.lanacion.com. ar/1811824.

La Nación (5 de julio de 2015a). Elecciones porteñas 2015: amplio triunfo de Horacio Rodríguez Larreta, pero habrá ballotage con Martín Lousteau. La Nación. Recolectado de: http:// www.lanacion.com.ar/1807919.

Medús, N. (1997). Los estudios electorales en la geografía. Huellas. 1, 9-25.

Medús, N. (2002). Voto, representación y territorio. Análisis y tendencias en la escala local. El municipio en la Provincia de La Pampa, Argentina. (Tesis doctoral, Universidad Nacional de Cuyo, Mendoza, Argentina).

Monzón, N. (2009a). Conceptos referidos al estudio electoral desde la ciencia geográfica. Geográfica Digital. 6(12). 
Monzón, N. (2009b). Geografía Electoral. Consideraciones teóricas para el caso argentino. Cuadernos de Geografía. Revista Colombiana de Geografía. 18, 119-128.

Obarrio, M. (2015). Randazzo criticó a Cristina por elegir a Scioli y quedó cerca de la renuncia. La Nación. Recuperado de: http://www.lanacion.com.ar/1841650.

Palma, E. \& Morales, G. (2014). La Geografía de las campañas presidenciales en México en 2012. América Latina Hoy. 67, 191-209.

Ramos, V. (2015). Martín Lousteau será el próximo Jefe de Gobierno de la Ciudad de Buenos Aires. Diario Perfil. Recuperado de: http://www.prefil.com/politica/martin-lousteausera-el-proximo-jefe-de-gobierno-de-la-diudad-de-buenos-aires.

Taylor, P. \& Johnston, R. (1979). Geography of elections. London: Penguin Books.

Velázquez, G. (1989). Geografía de las elecciones argentinas. Tandil: Universidad Nacional del Centro de la Provincia de Buenos Aires.

Velázquez, G. (2016). Geografía y Calidad de Vida en Argentina. Análisis regional y departamental 2010. Tandil: Universidad Nacional del Centro de la Provincia de Buenos Aires. 